\title{
Security and Efficiency of Percutaneous Microwave Ablation using Combined Computed Tomography and Ultrasound-Guided Imaging in Patients with Hepatocellular Carcinoma: A Prospective Study
}

\section{Wenpeng Zhao ( $\sim$ zwp215@163.com )}

Beijing Ditan Hospital

\section{Jiang Guo}

Capital Medical University Affiliated Beijing Ditan Hospital

\section{Honglu Li}

Capital Medical University Affiliated Beijing Ditan Hospital

\section{Liang Cai}

Capital Medical University Affiliated Beijing Ditan Hospital

\section{Youjia Duan}

Capital Medical University Affiliated Beijing Ditan Hospital

\section{Xiaopu Hou}

Capital Medical University Affiliated Beijing Ditan Hospital

Hongliu Du

Capital Medical University Affiliated Beijing Ditan Hospital

\section{Xihong Shao}

Capital Medical University Affiliated Beijing Ditan Hospital

\section{Zhenying Diao}

Capital Medical University Affiliated Beijing Ditan Hospital

\section{Changqing Li}

Capital Medical University Affiliated Beijing Ditan Hospital

\section{Research}

Keywords: Microwave ablation, hepatocellular carcinoma, computed tomography, ultrasound, Barcelona clinic liver cancer stage A

Posted Date: June 1st, 2020

DOI: https://doi.org/10.21203/rs.3.rs-31844/v1 
License: (c) (i) This work is licensed under a Creative Commons Attribution 4.0 International License. Read Full License 


\section{Abstract}

Background: We prospectively evaluated the security and efficiency of percutaneous microwave ablation (MWA) using combined computed tomography (CT) and ultrasound (US)-guided imaging in patients with BCLC-A1-3 hepatocellular carcinoma (HCC) given that they have shortcomings when used alone.

Methods: We included 88 consecutive patients with single HCC who were treated with transcatheter hepatic arterial chemoembolization (TACE). The patients were divided into 3 groups at random by using draw lots 1 week after TACE. The combination group (34 patients) received MWA under the guidance of CT and US, while the single group (CT group, 30 patients; US group, 24 patients) received MWA under the guidance of CT or US alone. Contrast-enhanced MRI or CT scans were performed in all patients 1, 3, 6 and 12 months after the procedure. The study endpoints included the treatment time, puncture time, local recurrence rate, and adverse events.

Results: The median diameter of the lesions was $3.1(1.5-4.2) \mathrm{cm}$. The median treatment time was 38.6 (30-45) min and 36.7 (30-47) $\mathrm{min}$ in the combination group and US group, respectively. The median puncture number was 1.2 (1-2) times and 1.1 (1-2) times, respectively. Both were significantly less than in the CT group (45.8 min and 4.2 times). The local recurrence rate was $5.9 \%$ in the combination group, which was significantly inferior to that in the US group (16.7\%). The grade $\mathrm{C}$ complication rate in the combination group was $5.9 \%$, while it was $13.3 \%$ and $8.3 \%$ in the CT group and US group, respectively. There was a statistically significant difference between the combination group and CT group.

Conclusions: Using CT- and US-guided microwave ablation in patients with BCLC-A1-3 hepatocellular carcinoma appeared to be much better in terms of security and efficiency than the use of microwave ablation under the guidance of CT or US alone.

\section{Background}

Percutaneous microwave ablation (MWA) is widely used in the treatment of hepatocellular carcinoma (HCC), and leads to a satisfactory 1-5-year overall survival, low local recurrence rate, and few adverse events $[1,2]$. In the majority of cases, percutaneous MWA is performed under imaging guidance such as computed tomography (CT) or ultrasound (US). However, each imaging guidance approach has its own advantages and disadvantages. US has the advantages of the possibility of real-time monitoring, is easyto-use, radiationless, has a low cost, and a clear display of blood vessels and the bile duct is possible; however, there are a few disadvantages such as low image resolution, low location accuracy, susceptibility to nearby organs (for instance, the ribs, lungs, and gastrointestinal tract), and difficulty evaluating the ablation range $[3,4]$. In contrast, CT can greatly avoid such disadvantages $[5,6]$. However, the disadvantages of CT are: it lacks real-time and dynamic tracking, has radiation hazards, is relatively expensive, and does not bring into evidence the vasculature. The disadvantages of each type of imaging guidance and monitoring will result in substantial limitations of MWA and will inevitably influence the safety and effectiveness of MWA. 
Previous research has mainly focused on single image-guided MWA with either US or CT for the treatment of HCC. As none of the imaging guidance methods alone is effective enough to treat $\mathrm{HCC}$, there might be a better effect with a combination of the two. In this study, from the perspective of technological success, we combined US and CT to explore whether we can maximally overcome the shortcomings, while improving the inadequacies of the use of CT or US guidance alone to obtain better results.

\section{Methods}

\section{Patients}

The study protocol was approved by the ethical committee of the Beijing Ditan Hospital. All patients signed an informed consent form before the procedure. A total of 88 patients with single Barcelona clinic liver cancer (BCLC)-A HCC eligible for MWA and admitted in the Beijing Ditan Hospital, Capital Medical University from November 1, 2017 to February 28, 2019 were enrolled in the study. The diagnostic criteria of HCC were assessed according to the guidelines for the diagnosis and treatment of primary liver cancer in the BCLC Staging Classification [7].

The inclusion criteria were as follows: (1) patients aged 18-80 years; (2) tumor clinical stage BCLC-A1-3: diameter $\leq 5 \mathrm{~cm}$, liver function Child-Pugh class A or B; no vascular cancer embolus, vascular and intrahepatic bile duct invasion, or distant metastases; (3) patients who did not receive any anticancer treatment such as surgery, radiotherapy, chemotherapy, ablation, or targeted drugs; and (4) the performance status score of patients was less than 2 , with no serious organ dysfunction syndrome such as heart, brain, liver, or kidney problems.

The exclusion criteria were as follows: (1) severe liver malfunction (Child-Pugh score $>9$, serum total bilirubin level $>3 \mathrm{mg} / \mathrm{dl}$, and prothrombin time-international normalized ratio $>1.5$ ); (2) severe hepatic atrophy, expected ablated area larger than one-third of liver volume; (3) patients with esophageal or gastric variceal bleeding in the last six months; (4) active infection or intrahepatic bile duct dilation; (5) uncorrectable coagulopathy (PLT<30×109/L, PT>30 s, PTA<40\%); (6) lesion adjacent to the diaphragm, gallbladder, and major vessel, or protruding liver surface; and (7) obstinate massive ascites and hepatic encephalopathy.

\section{Equipment}

We used a KV2100 microwave tumor treatment device (Nanjing Kangyou Microwave Energy Sources Institute, China; frequency, $2450 \mathrm{MHz}$; needle type, internal water-cooling; electrode diameter, 15G; electrode length, 150 or $180 \mathrm{~mm}$; power, $0-100 \mathrm{~W}$; and distance from the aperture of the MW emission to the needle tip, $11 \mathrm{~mm}$ ); the ultrasound machine was LOGIQ P6 (GE, USA), using a broadband convex array probe (frequency, 1-5 MHz); and the CT device was produced by Germany's Siemens AG (tube voltage, $120 \mathrm{kV}$; tube current, $200 \mathrm{~mA}$; slice thickness, $5 \mathrm{~mm}$; and pitch, 1). 


\section{Treatment}

All patients were initially treated with transcatheter arterial chemoembolization (TACE). The purpose of TACE was to interdict the tumor target artery and make the tumor easily-recognizable on CT images, and to reduce heat deposition resulting from the artery and enhance the efficiency and effects of MWA. The treatment process was as follows: hepatic artery angiography was performed using the Seldinger technique. Femoral arterial catheterization was conducted through the common hepatic artery or proper hepatic artery, and the location, number, size, and blood supply of the lesions were evaluated.

Subsequently, a microcatheter was super-selectively inserted into the hepatic lobe or hepatic segmental artery branch, and mixed suspensions of iodized oil (5-10 ml) and loplatin injection (40 mg) were infused into the artery through the catheter. Finally, blank microspheres $(100-300 \mu \mathrm{m})$ were infused to embolize the artery until the arterial blood flow supplying the tumor was completely blocked.

MWA was initiated 1 week after TACE. Patients were divided into 3 groups at random by using draw lots: the CT group, US group, and combination group. The procedure was performed under local anesthesia, and vital signs were monitored using an electrocardiogram. The patient was given a pethidine hydrochloride injection and diazepam injection 30 minutes before treatment. The microwave therapy instrument was in good working condition. Procedures were performed by one of two doctors with 10 years of experience in $\mathrm{HCC}$ ablation.

In the CT group, most patients were placed in the supine position, and a few patients were placed in the lateral decubitus position or prone position according to the point and direction of the embedded microwave electrode. CT scans provided clinically useful information such as the size, shape and position of the lesion, and the relationship between the lesion and adjacent structure; the distance from the lesion to the skin was measured, and the puncture path and site were confirmed. The skin around the puncture site was disinfected routinely, local anesthesia with lidocaine was used, a prepared guide pin $(21 \mathrm{G})$ was inserted in advance, and the position of the guide pin was dynamically adjusted according to the CT scanning image, enabling it to reach the edge of the lesion. Subsequently, the microwave electrode was inserted precisely into the lesions in the direction of the guide pin, the guide pin was removed, and the microwave electrode was adjusted slightly to the best position according to the CT scanning image. Microwave electrode placement was performed based on the expected ablation zone size described by the manufacturer, considering a sufficient $(>5 \mathrm{~mm}$ ) safety margin around the tumor. The microwave power was set at 50-60 W. The ablation time for each lesion was 5-8 minutes, and the ablation area covering the lesion and its surrounding area measured $5 \mathrm{~mm}$ or more. If a single treatment did not produce satisfactory results, the microwave electrode was adjusted according to the CT scanning image, and a second MWA treatment was conducted immediately until the ablation area covered the lesion. Routine ablation needle tracking was performed to prevent implantation metastasis, a pressure dressing was placed to prevent hemorrhage immediately after the procedure, and a postoperative CT scan was performed to confirm whether any complications (for example, pneumothorax, pleural effusion, subcapsular hemorrhage) required further management. After treatment, liver protection, anti- 
inflammatory, and sedation therapies were prescribed. A follow-up study with repeat contrast-enhanced MRI or CT was conducted, as shown in Fig 1.

In the US group, all patients were in supine or left lateral decubitus position following the principle that the lesions were more apparent in ultrasonic imaging. If necessary, artificial pleural effusion and ascites were used to treat US-invisible HCC in the hepatic dome or adjacent gastrointestinal tract before the procedure. Microwave electrodes were inserted precisely into the lesions under US guidance. The ablation power was 50-60 W, and the ablation time was 5-8 minutes. During the course of treatment, changes in the internal echoes of the lesion and manifestations of the intrahepatic and perihepatic tissues were observed by US in real time. When hyperechoic regions completely covered the targeted lesions, the therapy was stopped. The same needle track ablation and pressure dressing were performed after the procedure. Whether there were any complications such as pleural effusion and subcapsular hemorrhage were evaluated by US after treatment. Patients were regularly followed up for more than 12 months, as shown in Fig 2.

In the combination group at first, all patients underwent CT examination in a supine position. The best puncture path and site were designed according to the CT image, avoiding nearby larger blood vessels and the bile duct, pulmonary tissue and pleural cavity. Subsequently, the microwave electrode was inserted precisely into the lesions to avoid nearby larger blood vessels and the bile duct under real-time US guidance. Then, repeat CT was performed to further precisely target the position of the microwave electrode, and the relationship between the microwave electrode and the surrounding structure of the lesion when necessary, was slightly adjusted. The ablation power was 50-60 W, and the ablation time was 5-8 minutes. Internal echo changes in the lesions were observed by real-time US, and timely CT examination was performed. The therapy was stopped when the ablation area completely covered the targeted lesions and when there were no complications according to the CT image. Routine needle track ablation and pressure dressing were performed after the procedure. Regular follow-up examinations continued for more than 12 months, as shown in Fig 3.

\section{Efficacy and safety}

All patients underwent contrast-enhanced MRI or CT preoperatively as well as after 1, 3, 6, and 12 months of treatment. The treatment time, puncture time, and local recurrence rate were used to evaluate the efficacy of the three groups. During the follow-up period, if we found local recurrence, we treated the patients with a second MWA; if we found intrahepatic metastasis and distant metastasis, we treated the patients with other treatment methods such as targeted drugs.At the same time, the liver and kidney functions and AFP were recorded. The MWA-related complications including bile duct injury, gastrointestinal bleeding, hydrothorax, sepsis, liver failure, renal dysfunction, peritoneal hemorrhage, and skin burn were assessed.

\section{Statistical analyses}


Parameters were tested for normality using the Shapiro-Wilk test. The means and standard deviations (SDs) of continuous, normally distributed parameters were determined and compared using the one-way analysis of variance or independent samples $t$ test. Patient age, lesion size, treatment time, complete ablation rate, local recurrence rate, number of punctures, and adverse events were compared between the three groups. Differences with $p$ values $<0.05$ were considered significant, and $p$ values were not adjusted for multiple comparisons. Statistical analyses were performed with SPSS 19.0 software (SPSS, IBM Company, USA).

\section{Results}

\section{Patient characteristics}

The patients comprised 60 males and 28 females, with a mean age of $52 \pm 11.2$ (36-72) years. Fifty-six patients had elevated serum alpha-fetoprotein (AFP) levels. The combination group was made of 34 patients while the single group was made of 54 patients (CT group: 30 patients, US group: 24 patients).

\section{Operative Procedures}

All the procedures were performed according to the preoperative plan. A total of 88 lesions in 88 patients who were treated with TACE were radically treated with MWA. The baseline characteristics of the patients were shown in Table 1, and there were no significant differences among the three groups. The median diameter of the lesions was $3.1(1.5-4.2) \mathrm{cm}$. The median treatment time was $38.6(30-45) \mathrm{min}$ in the combination group, 45.8 (35-56) $\mathrm{min}$ in the CT group, and $36.7(30-47) \mathrm{min}$ in the US group. The mean puncture number was $1.2(1-2)$ times in the combination group, $4.2(3-7)$ times in the CT group, and 1.1 $(1-2)$ times in the US group. The treatment time and the mean puncture number in the combination group and US group were significantly better than those in the CT group. The details are shown in Table 2. 
Table 1

Baseline characteristics of patients with HCC

\begin{tabular}{|c|c|c|c|c|}
\hline Variables & Combination group $(n=34)$ & $\begin{array}{l}\text { CT group } \\
(n=30)\end{array}$ & $\begin{array}{l}\text { US group } \\
(n=24)\end{array}$ & Total \\
\hline $\begin{array}{l}\text { Age } \\
\text { (years) }\end{array}$ & $\begin{array}{l}53 \pm 10.8 \\
(40-71)\end{array}$ & $\begin{array}{l}50 \pm 11.6 \\
(36-70)\end{array}$ & $\begin{array}{l}54 \pm 11.4 \\
(44-72)\end{array}$ & $\begin{array}{l}52 \pm 11.2 \\
(36-72)\end{array}$ \\
\hline Sex (male/female) & $24 / 11$ & $20 / 9$ & $16 / 8$ & $60 / 28$ \\
\hline $\begin{array}{l}\text { Tumor size } \\
(\text { mean } \pm S D, c m)\end{array}$ & $\begin{array}{l}3.4 \\
(1.8-4.4)\end{array}$ & $\begin{array}{l}3.0 \\
(1.3-3.9)\end{array}$ & $\begin{array}{l}3.2 \\
(1.7-4.0)\end{array}$ & $\begin{array}{l}3.1 \\
(1.5-4.2)\end{array}$ \\
\hline $\begin{array}{l}\text { Tumor location } \\
\text { (left/right, n) }\end{array}$ & $12 / 22$ & $11 / 19$ & $9 / 15$ & $32 / 56$ \\
\hline $\begin{array}{l}\text { AFP } \\
(\mathrm{ng} / \mathrm{ml})\end{array}$ & $\begin{array}{l}46.6 \\
(6.8-158.4)\end{array}$ & $\begin{array}{l}40.8 \\
(7.9-110.2)\end{array}$ & $\begin{array}{l}43.8 \\
(5.2-160.1)\end{array}$ & $\begin{array}{l}42.7 \\
(7.1-140.2)\end{array}$ \\
\hline $\begin{array}{l}\text { Viral hepatitis } \\
\text { (HBV/HCV) }\end{array}$ & $32 / 2$ & $29 / 1$ & $23 / 1$ & $84 / 4$ \\
\hline $\begin{array}{l}\text { Cirrhosis } \\
(\%)\end{array}$ & 88.2 & 83.3 & 87.5 & 86.4 \\
\hline $\begin{array}{l}\mathrm{Hb} \\
(\mathrm{g} / \mathrm{L})\end{array}$ & $\begin{array}{l}121.2 \\
(93.8-148.7)\end{array}$ & $\begin{array}{l}131.0 \\
(102.5-152.1)\end{array}$ & $\begin{array}{l}120 \\
(100.6-154.3)\end{array}$ & $\begin{array}{l}124 \\
(108.8-144.6)\end{array}$ \\
\hline $\begin{array}{l}\text { PLT } \\
\left(\times 10^{9}\right)\end{array}$ & $\begin{array}{l}103.2 \\
(58.2-180.7)\end{array}$ & $\begin{array}{l}118.2 \\
(56.8-171.5)\end{array}$ & $\begin{array}{l}110.2 \\
(62.4-163.2)\end{array}$ & $\begin{array}{l}106.2 \\
(64.8-146.5)\end{array}$ \\
\hline $\begin{array}{l}\text { WBC } \\
\left(\times 10^{9}\right)\end{array}$ & $\begin{array}{l}4.2 \\
(2.7-6.8)\end{array}$ & $\begin{array}{l}5.1 \\
(2.2-7.3)\end{array}$ & $\begin{array}{l}4.7 \\
(2.4-7.0)\end{array}$ & $\begin{array}{l}4.6 \\
(2.5-6.4)\end{array}$ \\
\hline $\begin{array}{l}\text { ALB } \\
(\mathrm{g} / \mathrm{L})\end{array}$ & $\begin{array}{l}42.6 \\
(32.3-46.8)\end{array}$ & $\begin{array}{l}44.5 \\
(33.7-48.2)\end{array}$ & $\begin{array}{l}41.5 \\
(34.4-45.9)\end{array}$ & $\begin{array}{l}43.3 \\
(34.1-45.8)\end{array}$ \\
\hline $\begin{array}{l}\text { ALT } \\
(\mathrm{U} / \mathrm{L})\end{array}$ & $\begin{array}{l}43.3 \\
(20.5-65.8)\end{array}$ & $\begin{array}{l}39.6 \\
(26.8-55.6)\end{array}$ & $\begin{array}{l}45.4 \\
(30.8-64.2)\end{array}$ & $\begin{array}{l}40.9 \\
(29.6-60.8)\end{array}$ \\
\hline $\begin{array}{l}\text { TBIL } \\
\text { (umol/L) }\end{array}$ & $\begin{array}{l}19.1 \\
(10.2-28.8)\end{array}$ & $\begin{array}{l}17.2 \\
(8.2-30.3)\end{array}$ & $\begin{array}{l}20.5 \\
(12.4-33.5)\end{array}$ & $\begin{array}{l}19.6 \\
(10.5-27.4)\end{array}$ \\
\hline
\end{tabular}




\begin{tabular}{|lllll|}
\hline Variables & Combination group $(\mathbf{n = 3 4 )}$ & $\begin{array}{l}\text { CT group } \\
(\mathbf{n}=\mathbf{3 0})\end{array}$ & $\begin{array}{l}\text { US group } \\
(\mathbf{n}=\mathbf{2 4})\end{array}$ & Total \\
\hline PT & 12.1 & 12.7 & 12.6 \\
$(\mathrm{~s})$ & $(10.4-14.1)$ & $(10.1-13.8)$ & $(10.6-14.0)$ & $(10.5-13.7)$ \\
\hline \multicolumn{2}{l}{ There was no significant difference in all variables among the 3 groups. } \\
\hline
\end{tabular}

Table 2

A comparison of operation data among the 3 groups.

\begin{tabular}{|llll|}
\hline Variables & Combination group $(\mathbf{n}=\mathbf{3 4})$ & $\begin{array}{l}\text { CT group } \\
(\mathbf{n = 3 0 )}\end{array}$ & $\begin{array}{l}\text { US group } \\
(\mathbf{n}=\mathbf{2 4})\end{array}$ \\
\hline Treatment time & $38.6 \square$ & $45.8 \square$ & $36.7 \square$ \\
$(\mathrm{min})$ & $(30-45)$ & $(35-56)$ & $(30-47)$ \\
\hline Puncture time & 1.2 & 4.2 & 1.1 \\
$(\mathrm{n})$ & $(1-2) \Delta$ & $(3-7) \Delta$ & $(1-2) \triangle$ \\
\hline $\mathbf{\square}_{p=0.036 ;} \square p=0.028 ; \Delta p=0.016 ; \triangle p=0.015$ & & \\
\hline
\end{tabular}

\section{Clinical efficacy}

The median follow-up period was 14 (12-20) months. During the follow-up period, 8 patients (combination group: 2 patients, CT group: 2 patients, US group: 4 patients) experienced local recurrence and received repeat MWA, and the local recurrence rates were $5.9 \%, 6.7 \%$, and $16.7 \%$ in the combination group, CT group, and US group, respectively. There was a significant difference between the combination group and US group in terms of the local recurrence rate, which was statistically indistinguishable between patients with CT and US-guided MWA. Four patients (combination group: 1 patient, CT group: 2 patients, US group: 1 patient) had intrahepatic and distant metastases after MWA, and no significant difference was found among the three groups. There were no deaths during the follow-up period. The details are shown in Table 3. There was a significant reduction in the level of AFP among the three groups after treatment, but the rate of decline was not statistically significant among the three groups (Table 4). 
Table 3

\begin{tabular}{|c|c|c|c|}
\hline Variables & Combination group $(n=34)$ & $\begin{array}{l}\text { CT group } \\
(n=30)\end{array}$ & $\begin{array}{l}\text { US group } \\
(n=24)\end{array}$ \\
\hline Local recurrence rate (n/\%) & $2 / 5.9 \Delta$ & $2 / 6.7$ & $4 / 16.7^{\Perp}$ \\
\hline $\begin{array}{l}1 \text { mon post- } \\
\text { MWA (n/\%) }\end{array}$ & $0 / 0$ & $0 / 0$ & $1 / 4.2$ \\
\hline $\begin{array}{l}3 \text { mon post- } \\
\text { MWA (n/\%) }\end{array}$ & $1 / 2.9$ & $1 / 3.3$ & $2 / 8.3$ \\
\hline $\begin{array}{l}6 \text { mon post- } \\
\text { MWA (n/\%) }\end{array}$ & $1 / 2.9$ & $1 / 3.3$ & $1 / 4.2$ \\
\hline $\begin{array}{l}12 \text { mon post- } \\
\text { MWA (n/\%) }\end{array}$ & $0 / 0$ & $0 / 0$ & $0 / 0$ \\
\hline Grade C complication ( $\mathrm{n} / \%)$ & $2 / 5.9 \triangle$ & $4 / 13.3^{\triangle}$ & $2 / 8.3$ \\
\hline $\begin{array}{l}\text { Hyperpyrexia } \\
(\mathrm{n} / \%)\end{array}$ & $1 / 2.9$ & $1 / 3.3$ & $1 / 4.2$ \\
\hline $\begin{array}{l}\text { Hyperemesis } \\
(\mathrm{n} / \%)\end{array}$ & $1 / 2.9$ & $0 / 0$ & $0 / 0$ \\
\hline $\begin{array}{l}\text { Biloma } \\
(n / \%)\end{array}$ & $0 / 0$ & $1 / 3.3$ & $0 / 0$ \\
\hline Subcapsular hemorrhage $(n / \%)$ & $0 / 0$ & $1 / 3.3$ & $0 / 0$ \\
\hline $\begin{array}{l}\text { Hydrothorax } \\
(n / \%)\end{array}$ & $0 / 0$ & $1 / 3.3$ & $1 / 4.2$ \\
\hline
\end{tabular}

$\Delta_{p=0.038 ;}{ }^{\Delta} p=0.042$ 
Table 4

The variety of AFP in patients before and after treatment

\begin{tabular}{|c|c|c|c|c|c|}
\hline Variables & Basline & $\begin{array}{l}1 \text { mon post- } \\
\text { MWA }\end{array}$ & $\begin{array}{l}3 \text { mon post- } \\
\text { MWA }\end{array}$ & $\begin{array}{l}6 \text { mon post- } \\
\text { MWA }\end{array}$ & $\begin{array}{l}12 \text { mon post- } \\
\text { MWA }\end{array}$ \\
\hline $\begin{array}{l}\text { Combination } \\
\text { group } \\
(\mathrm{ng} / \mathrm{ml})\end{array}$ & $\begin{array}{l}46.6^{\Delta} \triangle \square \square \\
(6.8- \\
158.4 \rrbracket\end{array}$ & $\begin{array}{l}11.7^{凶} \\
(4.6-16.6 \rrbracket\end{array}$ & $\begin{array}{l}10.6^{\triangle} \\
(3.2-15.4 \rrbracket\end{array}$ & $\begin{array}{l}11.5 \square \\
(4.0-14.3 \rrbracket\end{array}$ & $\begin{array}{l}10.8^{\square} \\
(4.2-13.3 \rrbracket\end{array}$ \\
\hline $\begin{array}{l}\text { CT group } \\
(\mathrm{ng} / \mathrm{ml})\end{array}$ & $\begin{array}{l}40.8 \nabla \nabla 0 \square \\
(7.9- \\
110.2 \rrbracket\end{array}$ & $\begin{array}{l}9.9^{\nabla} \\
(3.6-18.2 \rrbracket\end{array}$ & $\begin{array}{l}11.5^{\nabla} \\
(3.8-16.6 \rrbracket\end{array}$ & $\begin{array}{l}10.4^{\square} \\
(3.4-15.7 \rrbracket\end{array}$ & $\begin{array}{l}11.7^{\square} \\
(3.9-15.3 \rrbracket\end{array}$ \\
\hline $\begin{array}{l}\text { US group } \\
\text { (ng/ml) }\end{array}$ & $\begin{array}{l}43.8000 \\
(5.2- \\
160.18\end{array}$ & $\begin{array}{l}12.8^{\bullet} \\
(4.4-17.4 \rrbracket\end{array}$ & $\begin{array}{l}11.8^{\bullet} \\
(3.9-16.6 \rrbracket\end{array}$ & $\begin{array}{l}10.9^{\square} \\
(4.1-15.8 \rrbracket\end{array}$ & $\begin{array}{l}11.9^{\square} \\
(4.2-15.2 \rrbracket\end{array}$ \\
\hline
\end{tabular}

$\triangle p=0.009 \rrbracket \Delta p=0.008 \rrbracket \square=0.009 \rrbracket \square p=0.007 \rrbracket p=0.006 \rrbracket \nabla p=0.008 \rrbracket^{\square} p=0.007 \rrbracket$

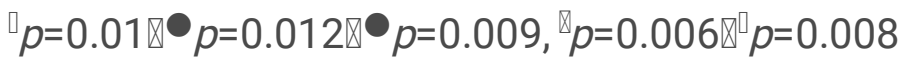

\section{Complications}

Complications were evaluated according to the unified standardized Society of Interventional Radiology (SIR) grading system [8]. The common adverse effects during treatment and after treatment were lowgrade fever, right epigastric mild pain, and mild nausea and vomiting, which commonly occurred 1-3 days after treatment; the majority of which belonged to grade A or B according to the SIR grading system and resolved spontaneously without any other treatment. Patients with grade $\mathrm{C}$ complications needed to be treated in the hospital.

The grade $\mathrm{C}$ complication rate in the combination group (hyperpyrexia: 1 patient, hyperemesis: 1 patient) was $5.9 \%$, while it was $13.3 \%$ and $8.3 \%$ in the CT group (hyperpyrexia: 1 patient, biloma: 1 patient, subcapsular hemorrhage: 1 patient, hydrothorax: 1 patient) and US group (hyperpyrexia: 1 patient, hydrothorax: 1 patient), respectively. There was a significant difference between the combination group and CT group $(p<0.05)$, and the difference was nonsignificant between the CT group and US group. All of the above-mentioned adverse effects were relieved by applying anti-inflammatory agents, analgesics, external drainage, hemostasia, and antiemetic drugs. The details are shown in Table 3.

A transient elevation in alanine aminotransferase (ALT) and total bilirubin (TBil) occurred, approaching the peak level on the 3rd day and returning to normal on the 7th day. Renal function showed no obvious changes postoperatively compared to preoperatively. 


\section{Discussion}

MWA can lead to complete necrosis in HCC; however, the cooling effect of blood flow has the greatest impact on the ablation zone, and the induced area of necrosis is small [9]. TACE has the advantage of reducing blood perfusion of tumors after hepatic arterial blockage. Therefore, it can decrease the cooling effect of blood flow on the heating action of MWA and enhance the coagulation action of MWA [10]. Combined therapy with TACE and MWA is safe and effective and has become a recommended treatment protocol in patients with HCC [11-13].

However, the limited literature comparing the thermal ablation of HCC under the guidance of CT and US has shown that both of these techniques have excellent outcomes [14]. It is worth noting that the postprocedural complication rates of thermal ablation vary greatly, from $2.2 \%$ to $53.2 \%[14,15]$; we cannot say that this is not influenced by the method of imaging guidance. Both types of imaging guidance have their own disadvantages; to overcome these limitations, various methods have been proposed by researchers to achieve this goal, such as CT/MRI-CEUS and US-CEUS fusion imaging techniques and multimodality imaging-compatible insertion robots [16-19]. All these measures can aid operators in performing technically challenging procedures, with the potential of reducing the procedure time and radiation dose. However, the calibration error caused by organ deformation, displacement, and respiratory movement cannot be avoided. The process is very complicated and time-consuming, and specific hardware and software requirements as well as trained professionals are needed. Further studies are also needed to clarify these problems [17-19].

In our analysis, we prospectively compared the effectiveness and safety of US, CT, and combinationguided percutaneous MWA in treating patients with HCC. The local recurrence and grade $\mathrm{C}$ complication rates were not significantly different between patients with CT and US-guided MWA, which was consistent with the findings of a previous related study [14,21]. US has the advantages of real-time monitoring, a short operation time, and a clear display of blood vessels and the bile duct, while CT has a high accuracy of puncture and a high image resolution [3,4]. Therefore, in the combination group, the operator could significantly shorten the treatment time, increase the success rate of puncture and reduce blood vessel and bile duct damage resulting from repeated puncture. This could explain why the treatment time, puncture time, and grade $\mathrm{C}$ complication rates in the combination group were inferior to those in the CT group. Theoretically, CT guidance for mass targeting leads to a certain degree of radiation exposure in patients. Therefore, reductions in treatment time and puncture time can dramatically reduce the radiation dose that harms patients [20]. One disadvantage of US guidance is the limited capability of visualizing tumor boundaries and monitoring thermal effects due to air bubbles produced by vaporization. CT guidance has a high accuracy and is not interrupted by air bubbles, particularly in patients with HCC who undergo TACE, which contributes to distinguishing adequate safety margins (measured as the distance from the initial tumor boundaries to the border of the posttreatment ablation zone) $[21,22]$. Therefore, these could partly explain why the local recurrence rates of the combination group were lower than those of the US group in our study. 
After treatment, AFP among the three groups continued to decline, and the liver and renal function showed no nonreversible damage compared to preoperative values. These results were consistent with past studies [23] demonstrating the validity and safety of MWA.

This study had limitations. The sample size was small. It was not a multicenter randomized controlled trial, and long-term outcomes were not investigated. However, preliminary results showed that combined guidance had an advantage over the use of a single imaging guidance. This leads to the need for further validation studies with long-term data. For some clinical variables that could potentially affect health outcomes such as tumor number, location, size, and comorbidity, we were unable to delve into these analyses to get the desired details. Therefore, more specific studies are needed in the future to compare MWA under different imaging guidance methods. We also included lesions less than $5 \mathrm{~cm}$ in size to assess the effects of MWA under different imaging guidance methods; the results were not comprehensive.

\section{Conclusions}

In conclusion, combined imaging guidance could overcome some of the disadvantages of CT and US when used individually for imaging guidance. CT- combined with US-guided microwave ablation in patients with BCLC-A1-3 HCC appeared to be much better in terms of security and efficiency than microwave ablation under the guidance of CT or US alone.

\section{Declarations}

\section{Ethics approval and consent to participate}

The study protocol was approved by the ethical committee of Beijing Ditan Hospital, Capital Medical University, the institute consent to participate.

\section{Consent for publication}

We confirm that all individual person in the study have consent to publish their data; The manuscript has been read and approved by all named authors and the order of authors listed in the manuscript has been approved by all of us. All authors have approved to submit to your journal for publication.

\section{Availability of data and material}

Please contact author for data requests

\section{Competing interests}

All authors declare that they have no any competing interest.

\section{Funding}


The subject is funded by the Scientific Research Foundation of Beijing Ditan Hospital (Grant No.DTYM201605)

\section{Authors' contributions}

Changqing Li, raised study concepts adn participated in study design. Wenpeng Zhao together with Jiang Guo performed the study.

Wenpeng Zhao drafted the manuscript.

Honglu Li,Liang Cai, Youjia Duan, Xiaopu Hou and Zhenying Diaocollected the clinical data and participated in statistical analysis.

Xihong Shao, Hongliu Du and Wei Li participated in quality control of data and algorithms.

\section{Acknowledgements}

The subject is supported by the Scientific Research Foundation of Beijing Ditan Hospital

\section{References}

1. Mrudula B Glassberg, Sudip Ghosh, Jeffrey W Clymer, Rana A Qadeer, Nicole C Ferko, Behnam Sadeghirad,et al. Microwave ablation compared with radiofrequency ablation for treatment of hepatocellular carcinoma and liver metastases: a systematic review and meta-analysis. Onco Targets Ther 2019; 12: 6407-6438.

2. Riccardo Lencioni, Laura Crocetti. Image-guided Thermal Ablation of Hepatocellular Carcinoma. Crit Rev Oncol Hematol 2008, 66:200-7.

3. Kim PN, Choi D, Rhim H, Rha SE, Hong HP, Lee J, et al. Planning ultrasound for percutaneous radiofrequency ablation to treat small $(<=3 \mathrm{~cm})$ hepatocellular carcinomas detected on computed tomography or magnetic resonance imaging: a multicenter prospective study to assess factors affecting ultrasound visibility. J Vasc Interv Radiol 2011, 23:627-634.

4. Jun Dong, Wang Li, Qi Zeng, Sheng Li, Xiao Gong, Lujun Shen, et al. CT-guided percutaneous step by step radiofrequency ablation for the treatment of carcinoma in the caudate lobe. Medicine 2015,94: e1594

5. Park BJ, Byun JH, Jin YH, Won HJ, Shin YM, Kim KW, et al. CT-guided radiofrequency ablation for hepatocellular carcinomas that were undetectable at US: therapeutic effectiveness and safety. $J$ Vasc Interv Radiol 2009, 20: 490-9.

6. Miura H, Yamagami T, Terayama K, Yoshimatsu R, Matsumoto T, Nishimura T. Pneumothorax induced by radiofrequency ablation for hepatocellular carcinoma beneath the diaphragm under realtime computed tomography- fluoroscopic guidance. Acta Radiol 2010,51:613-8. 
7. J M Llovet, C Brú, J Bruix. Prognosis of Hepatocellular Carcinoma: The BCLC Staging Classification. Semin Liver Dis 1999,19:329-38.

8. 8.Muneeb Ahmed, Luigi Solbiati, Christopher L. Brace, David J. Breen, Matthew R. Callstrom, J. William Charboneau, et al. Image-guided Tumor Ablation: Standardization of Terminology and Reporting Criteria-A 10-Year Update. Radiology 2014, 273: 241-260.

9. Nam C Yu , Steven S Raman, Young Jun Kim, Charles Lassman, Xinlian Chang, David S K Lu, et al. Microwave Liver Ablation: Influence of Hepatic Vein Size on Heat-Sink Effect in a Porcine Model. J Vasc Interv Radiol 2008,19:1087-92.

10. Higgins MCSS, Soulen MC. Combining Locoregional Therapies in the Treatment of Hepatocellular Carcinoma. Semin Interv Radiol 2013,30: 74- 81.

11. Wei-Zhu Yang, Na Jiang, Ning Huang, Jing-Yao Huang, Qu-Bin Zheng, Quan Shen, et al. Combined Therapy With Transcatheter Arterial Chemoem- bolization and Percutaneous Microwave Coagulation for Small Hepatocellular Carcinoma. World J Gastroenterol 2009,15:748-52.

12. Jian-ping Dou, Ping Liang, Jie Yu. Microwave ablation for liver tumors. Abdom Radiol 2016,41:650-8.

13. Maria Franca Meloni, Jason Chiang, Paul F Laeseke, Christoph F Dietrich, Angela Sannino, Marco Solbiati, et al. Microwave Ablation in Primary and Secondary Liver Tumours: Technical and Clinical Approaches. Int J Hyperthermia 2017,33:15-24.

14. Jinhai Huo, Thomas A Aloia, Ying Xu, Tong Han Chung, Tommy Sheu, Ya-Chen Tina Shih, et al. Comparative Effectiveness of Computed Tomography- Versus Ultrasound-Guided Percutaneous Radiofrequency Ablation Among Medicare Patients 65 Years of Age or Older with Hepatocellular Carcinoma. Value Health 2019,22:284-292.

15. 15.Tito Livraghi, Luigi Solbiati, M Franca Meloni, G Scott Gazelle, Elkan F Halpern, S Nahum Goldberg,et al. Treatment of Focal Liver Tumors with Percutaneous Radio-Frequency Ablation: Complications Encountered in a Multicenter Study. Radiology 2003,226:441-51.

16. Manoj Rajagopal , Aradhana M Venkatesan. Image Fusion and Navigation Platforms for Percutaneous Image-Guided Interventions. Abdom Radiol (NY)

17. 2016,41: 620-8.

18. Erjiao Xu, Yinglin Long, Kai Li, Qingjing Zeng, Lei Tan, Liping Luo, et al. Comparison of CT/MRI-CEUS and US-CEUS Fusion Imaging Techniques in the Assessment of the Thermal Ablation of Liver Tumors. Int J Hyperthermia 2019,135:159-167.

19. Xiao-Wan Bo, Hui-Xiong Xu, Dan Wang, Le-Hang Guo, Li-Ping Sun, Xiao-Long Li, et al. Fusion Imaging of Contrast-Enhanced Ultrasound and Contrast-Enhanced CT or MRI Before Radiofrequency Ablation for Liver Cancers.Br J Radiol 2016,89:20160379.

20. Dongrui Li, Zhigang Cheng, Gang Chen, Fangyi Liu, Wenbo Wu, Jie Yu, et al. A Multimodality Imaging-Compatible Insertion Robot With a Respiratory Motion Calibration Module Designed for Ablation of Liver Tumors: A Preclinical Study.Int J Hyperthermia 2018,34:1194-1201.

21. Dong Kyu Kim, Jong Yun Won, Sung Yoon Park. Percutaneous Cryoablation for Renal Cell Carcinoma Using Ultrasound-Guided Targeting and Computed Tomography-Guided Ice-Ball Monitoring: 
Radiation Dose and Short-Term Outcomes. Acta Radiol 2019,60:798-804.

22. Jing Wu, Ping Chen, Yang-gui Xie, Nian-mei Gong, Lin-lin Sun, Chun-feng Sun, et al. Comparison of the Effectiveness and Safety of Ultrasound- And CT-guided Percutaneous Radiofrequency Ablation of Non-Operation Hepatocellular Carcinoma. Pathol Oncol Res 2015,21:637-42.

23. Wei Teng, Ka-Wai Liu, Chen-Chun Lin, Wen-Juei Jeng, Wei-Ting Chen, I-Shyan Sheen, et al. Insufficient Ablative Margin Determined by Early Computed Tomography May Predict the Recurrence of Hepatocellular Carcinoma After Radiofrequency Ablation. Liver Cancer 2015,4:26-38.

24. Tian-Qi Zhang , Zhi-Mei Huang , Jing-Xian Shen, Gui-Qun Chen, Lu-Jun Shen, Fei Ai, et al. Safety and Effectiveness of Multi-Antenna Microwave Ablation-Oriented Combined Therapy for Large Hepatocellular Carcinoma. Therap Adv Gastroenterol 2019, 12:1756284819862966.

\section{Figures}

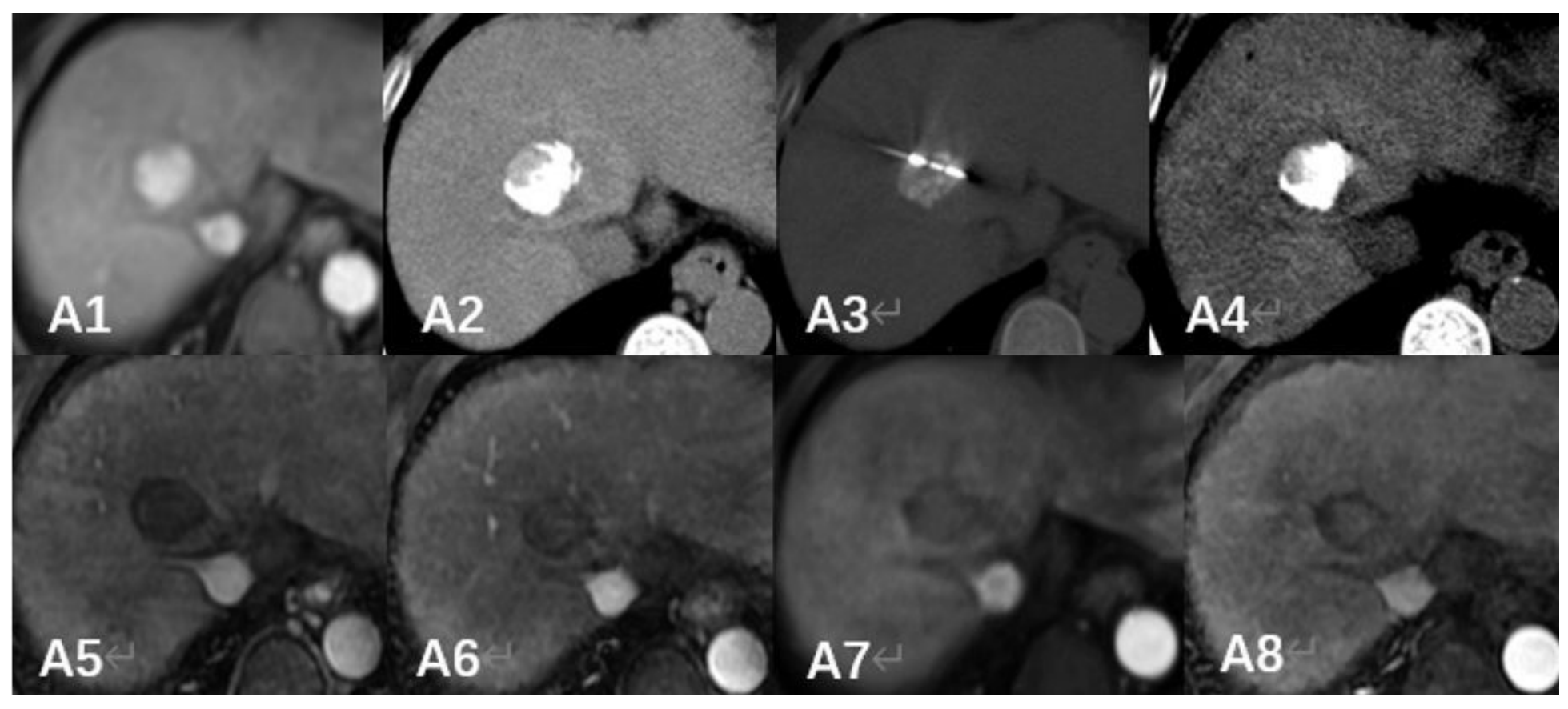

Figure 1

A 58-year-old female. A1: Contrast-enhanced MRI indicated a liver lesion located in segment 8, with a size of $2.8 \mathrm{~cm} \times 3.2 \mathrm{~cm}$; Arterial enhancement was observed. A2: After TACE, the accumulation of iodized oil in the lesion was satisfactory. A3, A4: MWA was applied under the guidance of CT; Around the lesion was low density ring after MWA on CT images. A5-A8: Subsequent contrast-enhanced MRI within 12 months confirmed that the lesion had been completely ablated (A5, 1 month post-MWA; A6, 3 months post-MWA; A7, 6 months post-MWA; A8, 12 months post-MWA). 


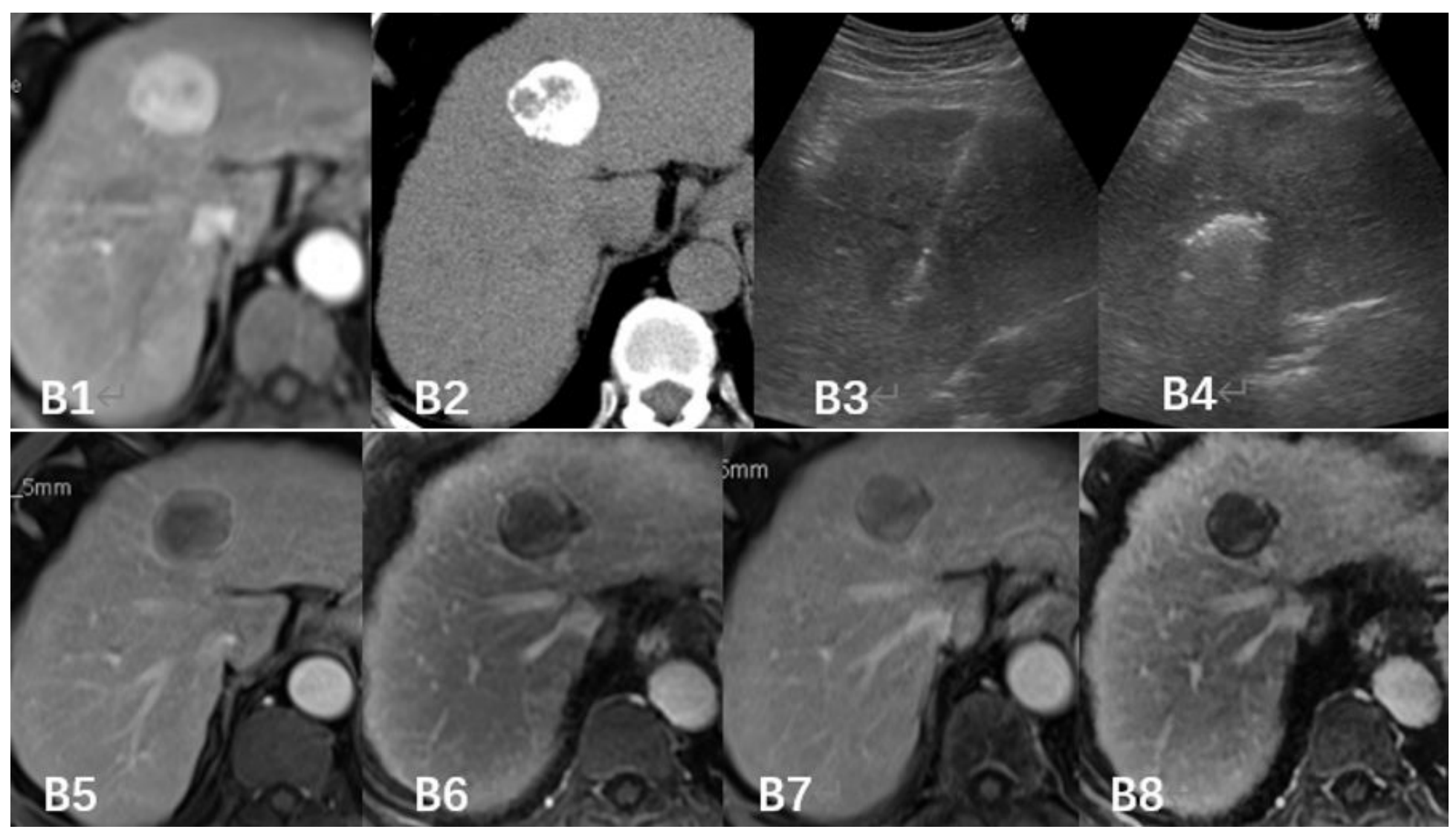

Figure 2

A 64-year-old male. B1: Contrast-enhanced MRI indicated a liver lesion located in segment 4, with a size of $3.6 \mathrm{~cm} \times 3.8 \mathrm{~cm}$; Arterial enhancement was observed. B2: After TACE, the accumulation of iodized oil in the lesion was satisfactory. B3, B4: A hypoechoic lesion in US, MWA was applied under the guidance and monitoring of real-time US; liver lesion was completely covered by diffusely increased echogenicity after MWA. B5-B8: Subsequent contrast-enhanced MRI within 12 months confirmed that the lesion had been completely ablated(B5, 1 month post-MWA; B6, 3 months post-MWA; B7, 6 months post-MWA; B8, 12 months post-MWA). 


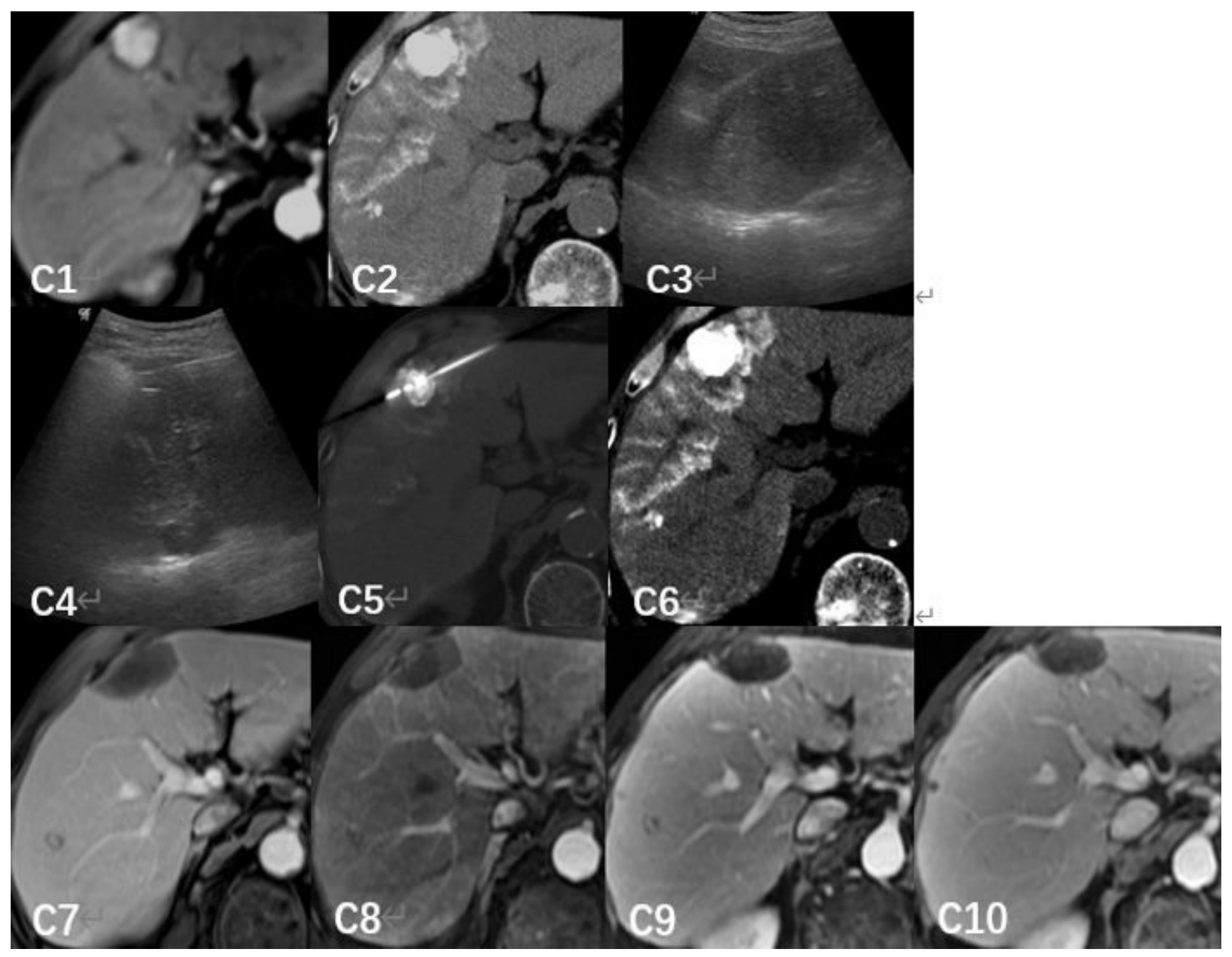

Figure 3

A 70-year-old male. C1: Contrast-enhanced MRI indicated a liver lesion located in segment 4, with a size of $2.6 \mathrm{~cm} \times 3.0 \mathrm{~cm}$; Arterial enhancement was observed. C2: After TACE, the accumulation of iodized oil in the lesion was satisfactory. C3, C4: Iso-hypoechoic lesion in US, MWA was applied under the guidance and monitoring of real-time US; Liver lesion was completely covered by diffusely increased echogenicity after MWA. C5, C6: Using CT scan further checked the position of Microwave electrode and evaluated whether the lesion had been completely ablated after MWA. C7-C10: Subsequent contrast-enhanced MRI within 12 months confirmed that the lesion had been completely ablated (C7, 1 month post-MWA; $\mathrm{C} 8,3$ months post-MWA; $\mathrm{C} 9,6$ months post-MWA; $\mathrm{C} 10,12$ months post-MWA). 